\title{
Cancer incidence and mortality in China, 2014
}

\section{Wanqing Chen, Kexin Sun, Rongshou Zheng, Hongmei Zeng, Siwei Zhang, Changfa Xia, Zhixun Yang, $\mathrm{He} \mathrm{Li,} \mathrm{Xiaonong} \mathrm{Zou,} \mathrm{Jie} \mathrm{He}$}

National Office for Cancer Prevention and Control, National Cancer Center/Cancer Hospital, Chinese Academy of Medical Sciences and Peking Union Medical College, Beijing 100021, China

Correspondence to: Jie He. National Office for Cancer Prevention and Control, National Cancer Center/Cancer Hospital, Chinese Academy of Medical Sciences and Peking Union Medical College, Beijing 100021, China. Email: prof.hejie@263.net; Wanqing Chen. National Office for Cancer Prevention and Control, National Cancer Center/Cancer Hospital, Chinese Academy of Medical Sciences and Peking Union Medical College, Beijing 100021, China. Email: chenwq@cicams.ac.cn.

\begin{abstract}
Background: National Central Cancer Registry of China (NCCRC) updated nationwide cancer statistics using population-based cancer registry data in 2014 collected from all available cancer registries.

Methods: In 2017, 449 cancer registries submitted cancer registry data in 2014, among which 339 registries' data met the criteria of quality control and were included in analysis. These cancer registries covered 288,243,347 population, accounting for about $21.07 \%$ of the national population in 2014 . Numbers of nationwide new cancer cases and deaths were estimated using calculated incidence and mortality rates and corresponding national population stratified by area, sex, age group and cancer type. The world Segi's population was applied for agestandardized rates.

Results: A total of 3,804,000 new cancer cases were diagnosed, the crude incidence rate was 278.07/100,000 (301.67/100,000 in males, 253.29/100,000 in females) and the age-standardized incidence rate by world standard population (ASIRW) was 186.53/100,000. Calculated age-standardized incidence rate was higher in urban areas than in rural areas $(191.6 / 100,000$ vs. 179.2/100,000). South China had the highest cancer incidence rate while Southwest China had the lowest incidence rate. Cancer incidence rate was higher in female for population between 20 to 54 years but was higher in male for population younger than 20 years or over 54 years. A total of 2,296,000 cancer deaths were reported, the crude mortality rate was $167.89 / 100,000(207.24 / 100,000$ in males, $126.54 / 100,000$ in females) and the age-standardized mortality rate by world standard population (ASMRW) was 106.09/100,000. Calculated age-standardized mortality rate was higher in rural areas than in urban areas (110.3/100,000 vs. 102.5/100,000). East China had the highest cancer mortality rate while North China had the lowest mortality rate. The mortality rate in male was higher than that in female. Common cancer types and major causes of cancer death differed between age group and sex.

Conclusions: Heavy cancer burden and its disparities between area, sex and age group pose a major challenge to public health in China. Nationwide cancer registry plays a crucial role in cancer prevention and control.
\end{abstract}

Keywords: Cancer registry; incidence; mortality; epidemiology; China

Submitted Jan 04, 2018. Accepted for publication Jan 29, 2018.

doi: 10.21147/j.issn.1000-9604.2018.01.01

View this article at: https://doi.org/10.21147/j.issn.1000-9604.2018.01.01

\section{Introduction}

Cancer burden is rising rapidly worldwide due to the aging of population and the adoption of new unhealthy lifestyle behaviors (1). Cancer is the leading cause of death and produces heavy disease burden in China (2). National cancer registry data with good quality and representativeness can reflect cancer burden of the country. In China, the 
national program of cancer registry was launched by the Ministry of Health in 2008. The National Central Cancer Registry of China (NCCRC) is responsible for collecting, evaluating and publishing national cancer statistics from local population-based cancer registries of China. In this paper, we reported nationwide cancer incidence and mortality in 2014, updating the overview of cancer burden in China. The statistical details of the scales and profiles of cancer in different areas, sexes and age groups can be used to effectively promote evidence-informed health policymaking in our country.

\section{Materials and methods}

\section{Cancer registry data source}

By 30th August 2017, 449 cancer registries from 31 provinces in China submitted cancer registry data of 2014 to NCCRC, of which 160 were located in cities above the county level and 289 were located in cities of the county level. All cancer registries covered 345,711,646 population (175,695,770 males and 170,015,876 females), accounting for about $25.27 \%$ of the national population at the end of 2014. All cancer cases were coded according to the International Classification of Diseases for Oncology, 3rd edition (ICD-O-3) and the International Statistical Classification of Diseases and Related Health Problems 10th Revision (ICD-10). Cancer registry data submitted to NCCRC underwent quality control based on the criteria of "Guideline for Chinese Cancer Registration" (3) and "Cancer Incidence in Five Continents Volume IX" by International Agency for Research on Cancer/International Association of Cancer Registries (IARC/IACR) (4). The validity, reliability, completeness and comparability of cancer registry data were evaluated based on a comprehensive consideration of a series of indexes including the mortality to incidence $(\mathrm{M} / \mathrm{I})$ ratio, the percentage of cases morphologically verified (MV) (\%), the percentage of death certificate-only cases (DCO) (\%), the percentage of the diagnosis of unknown basis (UB) (\%) and the stability of cancer trends over years. 339 cancer registries' data met the criteria of quality control and were included in pooled data, of which 129 were located in cities above the county level and 210 were located in cities of the county level (Figure 1). These cancer registries covered $288,243,347$ population $(146,203,891$ males and $142,039,456$ females), accounting for about $21.07 \%$ of the national population at the end of 2014 .

\section{National population data sources}

National Bureau of Statistics of the People's Republic of China provided national population data of 2014 stratified by area (urban/rural) and sex. We estimated age-specific population of 2014 using the fifth and sixth National Population Census data, which provided the whole population data of 2000 and 2010 by age group (0-, 1-4, $5-84$ by 5 years and $85+$ years). We firstly calculated the change of age-specific death probabilities between the fifth and sixth National Population Census data. Then we estimated the age-specific death probabilities of 2014 under the assumption of a linear interpolation and extrapolation, and estimated the population age structure of 2014 using these estimated death probabilities. Finally the age-specific population was calculated using the estimated age structure and the national population of 2014 released by the National Bureau of Statistics.

\section{Statistical analysis}

All cities/counties were classified into urban or rural areas according to the National Bureau of Statistics of the People's Republic of China. Crude cancer incidence and mortality rates in each stratum by age, sex, area (urban/rural) and region (seven administrative regions) were calculated using 339 cancer registries' data. We multiplied these incidence and mortality rates by estimated population in each stratum and added them up so as to obtain the estimated numbers of new cancer cases and deaths in China, 2014. The Segi's population was used for age-standardized rates (ASR). SAS software (Version 9.4, SAS Institute Inc., Cary, USA) was applied for statistical analysis.

\section{Results}

\section{Estimated numbers of new cancer cases and cancer incidence rates}

Table 1 displays the estimated nationwide numbers of new cancer cases, crude and age-standardized incidence rates of all cancers and 26 cancer types for male, female and both genders combined in 2014. About 3,804,000 new cancer cases were diagnosed in 2014, including 2,114,000 male cases and 1,690,000 female cases. The crude incidence rate was $278.07 / 100,000(301.67 / 100,000$ in males, $253.29 / 100,000$ in females). The age-standardized 


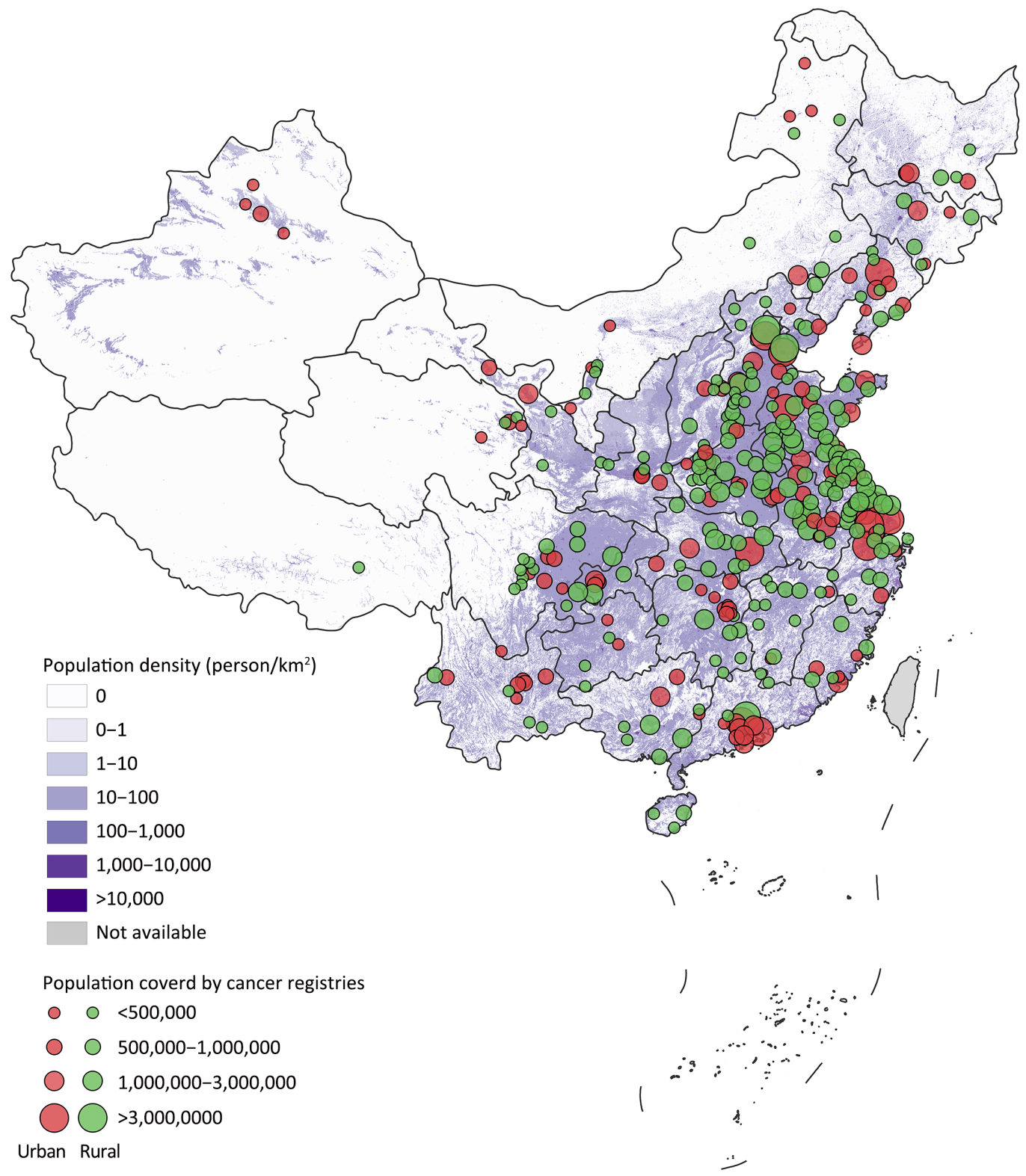

Figure 1 Map of 339 contributing cancer registries and geographic regions in China (the dots are locations of the cancer registries).

incidence rate by world standard population (ASIRW) was $186.53 / 100,000(208.33 / 100,000$ in males, $166.44 / 100,000$ in females). When considering cancer types, lung cancer was the most common cancer in whole population. Lung cancer was still the most common cancer in males and female breast cancer was the most common cancer in females (Table 1).

Table 2 displays the calculated age-standardized incidence rates (ASIR) of all cancers and selected major cancer types in different geographic areas in 2014. ASIR for all cancers combined was higher in urban areas than in rural areas (191.6/100,000 vs. 179.2/100,000). ASIRs for colorectal cancer, female breast cancer, prostate cancer, kidney cancer, bladder cancer, lymphoma and leukemia were higher in urban areas than in rural areas. ASIRs for esophageal cancer, stomach cancer, liver cancer and cervical cancer were higher in rural areas than in urban areas. ASIR for lung cancer in urban areas was similar to that in rural areas. South China had the highest cancer incidence rate $(200.6 / 100,000)$, followed by Northeast China $(190.2 / 100,000)$ and East China $(189.0 / 100,000)$. Southwest China $(165.8 / 100,000)$ had the lowest incidence 
Table 1 Estimated numbers of new cancer cases and incidence rates by sex in China, 2014

\begin{tabular}{|c|c|c|c|c|c|c|c|c|c|c|}
\hline \multirow[b]{2}{*}{ ICD-10 } & \multirow[b]{2}{*}{ Site } & \multicolumn{3}{|c|}{$\overline{A l l}$} & \multicolumn{3}{|c|}{ Male } & \multicolumn{3}{|c|}{ Female } \\
\hline & & $\begin{array}{l}\text { Cases } \\
\left(\times 10^{4}\right)\end{array}$ & $\begin{array}{c}\text { Crude } \\
\text { incidence } \\
\left(1 / 10^{5}\right)\end{array}$ & $\begin{array}{l}\text { ASIRW } \\
\left(1 / 10^{5}\right)^{*}\end{array}$ & $\begin{array}{l}\text { Cases } \\
\left(\times 10^{4}\right)\end{array}$ & $\begin{array}{c}\text { Crude } \\
\text { incidence } \\
\left(1 / 10^{5}\right)\end{array}$ & $\begin{array}{l}\text { ASIRW } \\
\left(1 / 10^{5}\right)^{\star}\end{array}$ & $\begin{array}{l}\text { Cases } \\
\left(\times 10^{4}\right)\end{array}$ & $\begin{array}{c}\text { Crude } \\
\text { incidence } \\
\left(1 / 10^{5}\right)\end{array}$ & $\begin{array}{l}\text { ASIRW } \\
\left(1 / 10^{5}\right)^{\star}\end{array}$ \\
\hline $\begin{array}{l}\text { C00-10, } \\
12-14\end{array}$ & $\begin{array}{l}\text { Lip, oral cavity \& } \\
\text { pharynx but } \\
\text { Nasopharynx }\end{array}$ & 4.6 & 3.35 & 2.29 & 3.1 & 4.41 & 3.10 & 1.5 & 2.24 & 1.49 \\
\hline C16 & Stomach & 41.0 & 30.00 & 19.51 & 28.8 & 41.08 & 27.94 & 12.3 & 18.36 & 11.27 \\
\hline C18-21 & Colorectum & 37.0 & 27.08 & 17.52 & 21.4 & 30.55 & 20.72 & 15.6 & 23.43 & 14.40 \\
\hline $\mathrm{C} 22$ & Liver & 36.5 & 26.67 & 17.81 & 26.9 & 38.37 & 26.74 & 9.6 & 14.38 & 8.86 \\
\hline $\mathrm{C} 23,24$ & Gallbladder & 5.2 & 3.82 & 2.37 & 2.5 & 3.59 & 2.38 & 2.7 & 4.05 & 2.36 \\
\hline C37, 38 & Other thoracic organs & 1.2 & 0.86 & 0.63 & 0.7 & 1.00 & 0.75 & 0.5 & 0.71 & 0.51 \\
\hline $\mathrm{C} 40,41$ & Bone & 2.4 & 1.76 & 1.32 & 1.4 & 1.95 & 1.51 & 1.0 & 1.55 & 1.13 \\
\hline $\mathrm{C} 43$ & Melanoma of skin & 0.7 & 0.49 & 0.34 & 0.4 & 0.50 & 0.36 & 0.3 & 0.49 & 0.33 \\
\hline C50 & Female breast & 27.9 & 41.82 & 28.77 & - & - & - & 27.9 & 41.82 & 28.77 \\
\hline C53 & Cervix & 10.2 & 7.46 & 5.26 & - & - & - & 10.2 & 15.30 & 10.61 \\
\hline C54, 55 & Uterus & 6.4 & 4.68 & 3.28 & - & - & - & 6.4 & 9.61 & 6.60 \\
\hline C56 & Ovary & 5.1 & 3.70 & 2.66 & - & - & - & 5.1 & 7.58 & 5.37 \\
\hline C61 & Prostate & 6.9 & 5.02 & 2.92 & 6.9 & 9.80 & 6.10 & - & - & - \\
\hline C62 & Testis & 0.3 & 0.23 & 0.20 & 0.3 & 0.45 & 0.39 & - & - & - \\
\hline C91-95 & Leukemia & 8.1 & 5.94 & 5.05 & 4.7 & 6.64 & 5.69 & 3.5 & 5.20 & 4.40 \\
\hline A_O & All other sites & 17.1 & 12.20 & 8.41 & 9.2 & 12.62 & 9.03 & 7.8 & 11.77 & 7.82 \\
\hline ALL & All sites & 380.4 & 278.07 & 186.53 & 211.4 & 301.67 & 208.33 & 169.0 & 253.29 & 166.44 \\
\hline
\end{tabular}

ICD-10, International Statistical Classification of Diseases and Related Health Problems 10th Revision; ${ }^{*}$, age-standardized incidence rate by world standard population; CNS, central nervous system.

rate. Lung cancer was the most common cancer in most regions except Northwest China, where stomach cancer was the most common cancer (Table 2).

\section{Estimated numbers of cancer deaths and cancer mortality rates}

Table 3 displays the estimated nationwide numbers of cancer deaths, crude and age-standardized mortality rates of all cancers and 26 cancer types for male, female and both genders combined in 2014. About 2,296,000 cancer deaths were reported in 2014, including $1,452,000$ male deaths and 844,000 female deaths. The crude mortality rate was $167.89 / 100,000$ (207.24/100,000 in males, 126.54/100,000 in females). The age-standardized mortality rate by world standard population (ASMRW) was 106.09/100,000 (138.34/100,000 in males, 75.15/100,000 in females). 
Table 2 Incidence rates of selected cancers by geographic areas in China, $2014\left(1 / 10^{5}\right)^{*}$

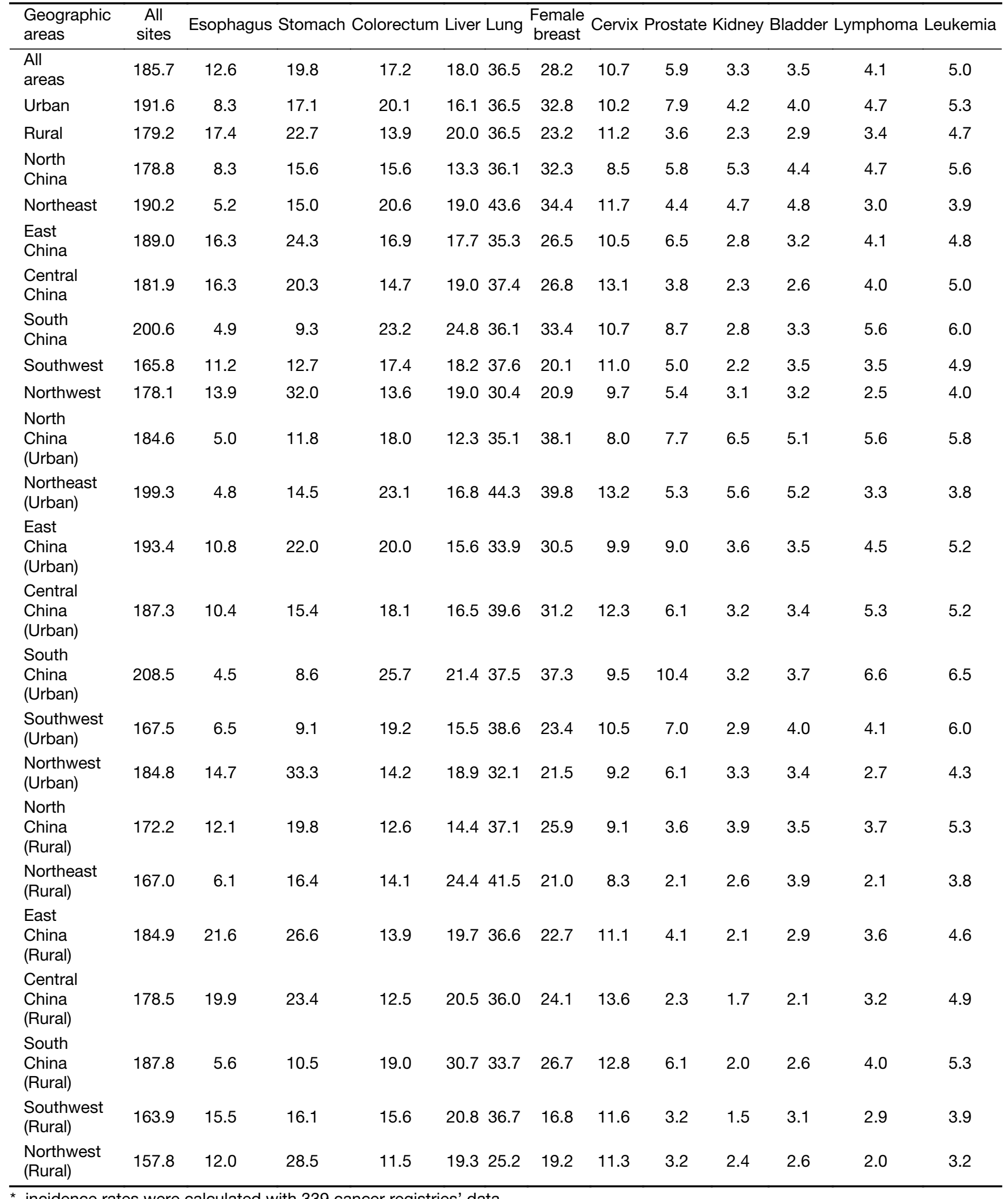

*, incidence rates were calculated with 339 cancer registries' data. 
Table 3 Estimated cancer deaths and mortality rates by sex in China, 2014

\begin{tabular}{|c|c|c|c|c|c|c|c|c|c|c|}
\hline \multirow[b]{2}{*}{ ICD-10 } & \multirow[b]{2}{*}{ Site } & \multicolumn{3}{|c|}{ All } & \multicolumn{3}{|c|}{ Male } & \multicolumn{3}{|c|}{ Female } \\
\hline & & $\begin{array}{c}\text { Deaths } \\
\left(\times 10^{4}\right)\end{array}$ & $\begin{array}{c}\text { Crude } \\
\text { mortality } \\
\left(1 / 10^{5}\right)\end{array}$ & $\begin{array}{c}\text { ASMRW } \\
\left(1 / 10^{5}\right)^{\star}\end{array}$ & $\begin{array}{l}\text { Deaths } \\
\left(\times 10^{4}\right)\end{array}$ & $\begin{array}{c}\text { Crude } \\
\text { mortality } \\
\left(1 / 10^{5}\right)\end{array}$ & $\begin{array}{l}\text { ASMRW } \\
\left(1 / 10^{5}\right)^{*}\end{array}$ & $\begin{array}{c}\text { Deaths } \\
\left(\times 10^{4}\right)\end{array}$ & $\begin{array}{c}\text { Crude } \\
\text { mortality } \\
\left(1 / 10^{5}\right)\end{array}$ & $\begin{array}{c}\text { ASMRW } \\
\left(1 / 10^{5}\right)^{\star}\end{array}$ \\
\hline C16 & Stomach & 29.4 & 21.48 & 13.30 & 20.5 & 29.24 & 19.21 & 8.9 & 13.33 & 7.63 \\
\hline C18-21 & Colorectum & 18.0 & 13.13 & 7.91 & 10.4 & 14.84 & 9.58 & 7.6 & 11.34 & 6.33 \\
\hline $\mathrm{C} 22$ & Liver & 31.9 & 23.31 & 15.29 & 23.4 & 33.32 & 22.98 & 8.5 & 12.78 & 7.62 \\
\hline $\mathrm{C} 23,24$ & Gallbladder & 3.9 & 2.86 & 1.71 & 1.8 & 2.59 & 1.67 & 2.1 & 3.14 & 1.74 \\
\hline C37, 38 & Other thoracic organs & 0.6 & 0.45 & 0.31 & 0.4 & 0.57 & 0.41 & 0.2 & 0.33 & 0.21 \\
\hline $\mathrm{C} 40,41$ & Bone & 1.7 & 1.26 & 0.86 & 1.0 & 1.44 & 1.03 & 0.7 & 1.06 & 0.69 \\
\hline C43 & Melanoma of skin & 0.4 & 0.27 & 0.17 & 0.2 & 0.26 & 0.17 & 0.2 & 0.27 & 0.16 \\
\hline C50 & Female Breast & 6.6 & 9.90 & 6.35 & - & - & - & 6.6 & 9.90 & 6.35 \\
\hline C53 & Cervix & 3.1 & 2.23 & 1.49 & - & - & - & 3.1 & 4.57 & 2.98 \\
\hline C54, 55 & Uterus & 1.6 & 1.20 & 0.78 & - & - & - & 1.6 & 2.46 & 1.54 \\
\hline C56 & Ovary & 2.3 & 1.64 & 1.11 & - & - & - & 2.3 & 3.37 & 2.22 \\
\hline C61 & Prostate & 3.0 & 2.16 & 1.13 & 3.0 & 4.22 & 2.46 & - & - & - \\
\hline C62 & Testis & 0.1 & 0.06 & 0.04 & 0.1 & 0.12 & 0.09 & - & - & - \\
\hline C91-95 & Leukemia & 5.2 & 3.78 & 2.88 & 3.0 & 4.31 & 3.34 & 2.2 & 3.22 & 2.43 \\
\hline A_O & All other sites & 9.3 & 6.67 & 4.27 & 5.3 & 7.35 & 5.00 & 4.0 & 5.95 & 3.57 \\
\hline ALL & All sites & 229.6 & 167.89 & 106.09 & 145.2 & 207.24 & 138.34 & 84.4 & 126.54 & 75.15 \\
\hline
\end{tabular}

ICD-10, International Statistical Classification of Diseases and Related Health Problems 10th Revision; *, age-standardized mortality rate by world standard population; CNS, central nervous system.

When considering cancer types, lung cancer was the most common cause of cancer deaths in both males and females (Table 3).

Table 4 displays the calculated age-standardized mortality rates (ASMR) of all cancers and selected major cancer types in different geographic areas in 2014. ASMR for all cancers combined was higher in rural areas than in urban areas (110.3/100,000 vs. 102.5/100,000). ASMRs for colorectal cancer, female breast cancer, prostate cancer, kidney cancer, bladder cancer and lymphoma were higher in urban areas than in rural areas. ASMRs for esophageal cancer, stomach cancer, liver cancer, lung cancer, cervical cancer and leukemia were higher in rural areas than in urban areas. East China had the highest cancer mortality rate (109.5/100,000), followed by Northeast China (108.5/ $100,000)$ and Central China $(108.5 / 100,000)$. North China 
Table 4 Mortality rates of selected cancers by geographic areas in China, $2014\left(1 / 10^{5}\right)^{*}$

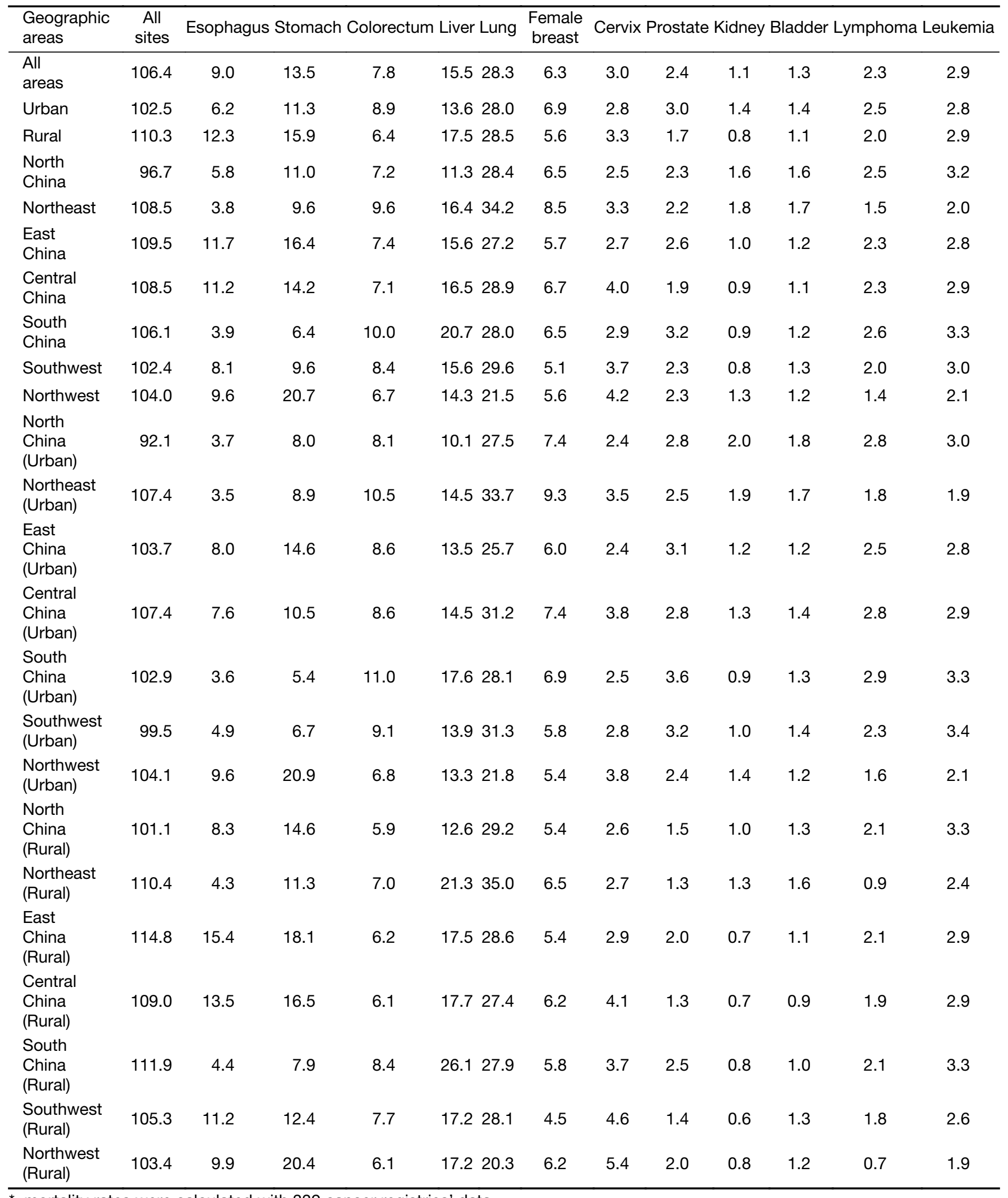

${ }^{\star}$, mortality rates were calculated with 339 cancer registries' data. 
(96.7/100,000) had the lowest mortality rate. Lung cancer was the leading cause of cancer death in all regions (Table 4).

\section{Age-specific incidence and mortality}

For both male and female, age-specific incidence rates were relatively low before 40 years and increased rapidly afterwards, reaching peak at the age group of 80-84 years and decreased slightly afterwards. Cancer incidence rates in female between 20 to 54 years were higher than that in male of the same age. Cancer incidence rates in male younger than 20 years or over 54 years were higher than that in female. The number of new cancer cases peaked at age group of 60-64 years both in male and female (Figure 2A).

For both male and female, age-specific mortality rates were low before 45 years and increased rapidly afterwards, reaching peak after 85 years. The mortality rates in male were consistently higher than that in female in all age groups. The number of deaths peaked at age group of 65-69 years in male and at age group of 75-79 years in female (Figure 2B).

\section{Estimated numbers of new cancer cases and cancer deaths by age, sex and cancer sites}

Table 5 displays top five estimated new cancer cases in different age groups by sex. In male, the five most common cancer types were lung cancer, stomach cancer, liver cancer, colorectal cancer and esophageal cancer. In female, the five most common cancer types were female breast cancer, lung cancer, colorectal cancer, thyroid cancer and stomach cancer. Major cancer types differed between age groups. The five most common cancer types in male and female aged between $0-14$ years were the same, which were leukemia, brain cancer, lymphoma, bone cancer and kidney cancer. Estimated new cases of thyroid cancer ranked second in male between 15-44 years. Lung cancer, liver cancer, stomach cancer, colorectal cancer and esophageal cancer were major cancer types in male between 15-80+ years. Estimated new cases of female breast cancer and thyroid cancer were both high in female aged 15-59 years. Estimated new cases of lung cancer increased rapidly with age and became the most common cancer in female after 60 years old. Colorectal cancer, stomach cancer, liver cancer and esophageal cancer were also common cancers in female after 60 years old (Table 5).

Table 6 displays top five estimated cancer deaths in different age groups by sex. In male, the five most common causes of cancer deaths were lung cancer, liver cancer, stomach cancer, esophageal cancer and colorectal cancer. In female, the five most common causes of cancer deaths were lung cancer, stomach cancer, liver cancer, colorectal cancer and female breast cancer. Major cancer causes differed between age groups. Common causes of cancer deaths in male and female aged between 0-14 years were the same, which were leukemia, brain cancer, lymphoma, liver cancer and bone cancer. Liver cancer, lung cancer, leukemia, stomach cancer and brain cancer were common

Table 5 Estimated five leading types of new cancer cases by age and sex in China, 2014 (thousands)

\begin{tabular}{|c|c|c|c|c|c|c|c|c|c|c|}
\hline Sites & All & Sites & 0-14 Sites & $15-44$ & Sites & $45-59$ & Sites & $60-79$ & Sites & $80+$ \\
\hline \multicolumn{11}{|l|}{ Male } \\
\hline All sites & $2,114.0$ & All sites & 12.9 All sites & 153.0 & All sites & 523.5 & All sites & 1160.8 & All sites & 263.9 \\
\hline Stomach & 287.9 & Brain, CNS & 2.5 Thyroid & 19.5 & Liver & 95.0 & Stomach & 177.0 & Stomach & 34.6 \\
\hline Liver & 268.9 & Lymphoma & 0.8 Lung & 15.6 & Stomach & 66.0 & Liver & 121.2 & Colorectum & 28.7 \\
\hline Esophagus & 185.4 & Kidney & 0.3 Stomach & 10.2 & Esophagus & 41.6 & Esophagus & 118.2 & Liver & 22.4 \\
\hline \multicolumn{11}{|l|}{ Female } \\
\hline All sites & $1,689.5$ & All sites & 9.5 All sites & 244.3 & All sites & 521.5 & All sites & 711.8 & All sites & 202.3 \\
\hline Breast & 278.9 & Leukemia & 3.8 Breast & 61.9 & Breast & 127.6 & Lung & 142.7 & Lung & 49.7 \\
\hline Lung & 260.7 & Brain, CNS & 1.7 Thyroid & 51.0 & Lung & 56.3 & Colorectum & 82.2 & Colorectum & 27.2 \\
\hline
\end{tabular}

CNS, central nervous system. 
A

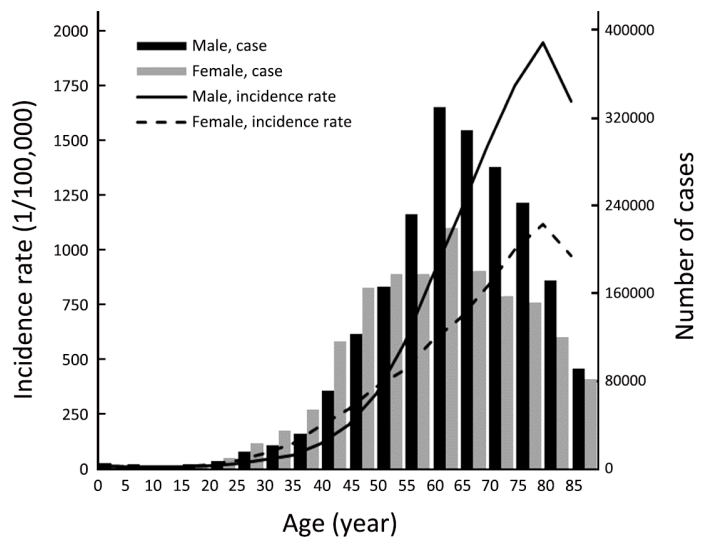

B

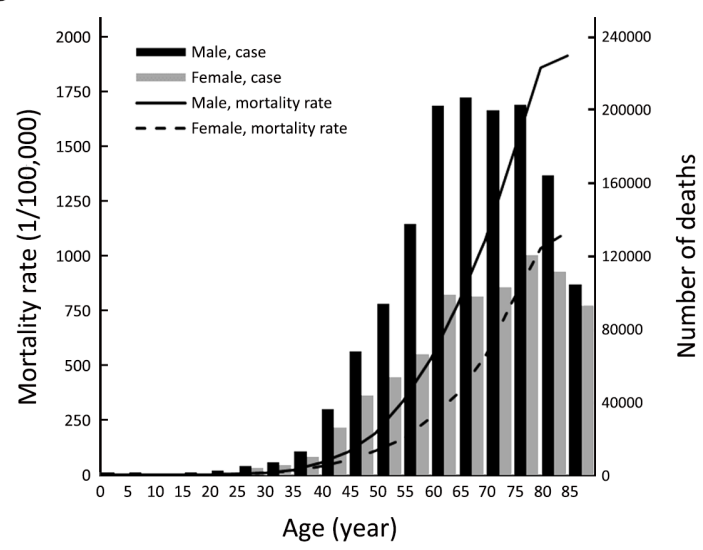

Figure 2 Age-specific cancer incidence and mortality by sex in China, 2014. (A) Age-specific cancer incidence rates and new cases by sex; (B) Age-specific cancer mortality rates and deaths by sex.

Table 6 Estimated five leading types of cancer deaths by age and sex in China, 2014 (thousands)

\begin{tabular}{|c|c|c|c|c|c|c|c|c|c|c|c|}
\hline Sites & All & Sites & $0-14$ & Sites & $15-44$ & Sites & $45-59$ & Sites & $60-79$ & Sites & $80+$ \\
\hline \multicolumn{12}{|l|}{ Male } \\
\hline All sites & $1,452.3$ & All sites & 5.1 & All sites & 67.2 & All sites & 299.4 & All sites & 812.1 & All sites & 268.5 \\
\hline Liver & 233.5 & Brain, CNS & 1.5 & Lung & 9.6 & Lung & 76.1 & Stomach & 126.0 & Stomach & 38.8 \\
\hline Stomach & 204.9 & Lymphoma & 0.3 & Leukemia & 5.4 & Stomach & 34.8 & Liver & 107.8 & Colorectum & 25.9 \\
\hline Colorectum & 104.0 & Bone & 0.2 & Brain, CNS & 4.4 & Colorectum & 18.0 & Colorectum & 55.8 & Esophagus & 24.3 \\
\hline \multicolumn{12}{|l|}{ Female } \\
\hline All sites & 844.1 & All sites & 3.7 & All sites & 50.2 & All sites & 164.1 & All sites & 421.4 & All sites & 204.7 \\
\hline Lung & 198.2 & Leukemia & 1.4 & Breast & 8.2 & Lung & 31.1 & Lung & 107.0 & Lung & 53.8 \\
\hline Stomach & 88.9 & Brain, CNS & 1.0 & Lung & 6.3 & Breast & 24.6 & Stomach & 46.5 & Stomach & 24.4 \\
\hline
\end{tabular}

CNS, central nervous system.

causes of cancer deaths in male aged 15-44 years. Liver cancer, lung cancer, stomach cancer, esophageal cancer and colorectal cancer were common causes of cancer deaths in male aged 45-80+ years. Female breast cancer and cervical cancer were both common causes of cancer deaths in female aged 15-59 years. Lung cancer, stomach cancer and liver cancer were common in female aged $15-80+$ years. Colorectal cancer and esophageal cancer were more common in female aged over 60 years (Table 6).

\section{Discussion}

In this paper, we updated cancer statistics in China in 2014 using eligible cancer registry data from 339 local cancer registries, representing $21.07 \%$ of the national population. The updated statistics provided a national profile of current cancer burden in China, creating scientific evidences for cancer prevention and control policymaking.

Cancer is the major public health problem and the leading cause of death worldwide. GLOBOCAN estimated that about 20 million new cancer cases were expected annually in 2025 (5). Latest cancer statistics report illustrated that 1.7 million new cancer cases and 0.6 million cancer deaths are projected to occur in 2018 in United States. It is inspiring to know that the cancer incidence rate and cancer death rate have been declining continuously in 
U.S. over the years, indicating that changes in behaviors and medical practice can be effective for cancer prevention and control (6). In China, cancer is the leading cause of death and disease burden of cancer is still rising (2,7-10). Thus, it remains a priority to develop targeted national cancer prevention and control plans and assess their effectiveness in China.

Cancer disease burden and cancer spectrum differed between regions in China, reflecting the geographical disparities in living habits and healthcare level. Common cancer types in urban areas were more similar to those in developed regions in the world, where the incidences of cancers relating to obesity and westernized life styles were high, including colorectal cancer, prostate cancer, kidney cancer and bladder cancer $(5,11)$. However, similarities were shared between rural areas and less developed regions in the world, where cancers relating to poverty and chronic infectious agents were more common, including esophageal cancer, stomach cancer, liver cancer and cervical cancer $(5,11)$. Higher mortality rate was found in rural areas, indicating poorer cancer prognosis due to late diagnosis and unsatisfying clinical treatment. The distinct disease patterns of different areas emphasize a need for precise cancer prevention and control plans customized for different geographical regions. For example, endoscopic screening program and Helicobacter Pylori eradication program launched in high risk areas of esophageal and gastric cancers in China obtained a profound positive effect on cancer control (12-15). However, the target population for upper gastrointestinal cancer screening programs should be precisely identified in non-high risk areas and the cost-effectiveness of such programs should be carefully evaluated (16).

Lung cancer was still the most commonly diagnosed cancer and the leading cause of cancer death in China. Lung cancer incidence and mortality rates in males were nearly twice as high as those in females, while no significant differences were found between different areas. Agestandardized 5-year survival for lung cancer was $16.1 \%$, for patients were normally diagnosed at late stages (17). Thus, it can be seen that the disease burden of lung cancer was still high in China. However, lung cancer incidence rate continues to decline in U.S., which can be mainly attributed to multi-level lung cancer prevention and screening programs (6). After decades of effort put into tobacco cessation programs, smoking prevalence in U.S. declined between 1996 and 2012 with the decline more significant in males than in females (18). What's more, there was strong evidence that low-dose computed tomography (LDCT) can reduce lung cancer mortality by up to $20 \%$ among current and former heavy smokers in U.S. (19). In 2013, the American Cancer Society began to recommend LDCT screening for individuals at high risk (20). U.S. experiences demonstrate that tobacco cessation and screening programs can efficiently lower lung cancer incidence, on which we can draw important lessons when facing the challenge of lung cancer in China.

Female breast cancer was the most commonly diagnosed cancer in female Chinese. However, its good prognosis led to a relatively low mortality rate, ranking it the fifth cause of cancer deaths in females. Female breast cancer disease burden has experienced a rapid growth over the last decade $(10,21,22)$. Rising socioeconomic status, westernized eating habits and living styles, obesity, reproductive factors and endocrine factors were important risk factors for female breast cancer, partly explaining the fact that the incidence and mortality rates were higher in urban areas than in rural areas $(23,24)$. It was noticeable that the incidence rate of female breast cancer ranked the highest in age groups 15-44 and 45-59 years. Independent studies reported that the age peak of disease onset was between $45-55$ years in China, which was earlier compared to western countries $(21,24-26)$. Thus the breast cancer screening programs in China should target at younger populations.

In the past decade, a series of cancer control programs have been launched in high risk areas of China, including cancer screening programs for esophageal cancer, stomach cancer, liver cancer, female breast cancer and cervical cancer. Since many of these programs have yielded significant benefits, Chinese government continues to expand its cancer screening network, now covering 231 counties in 31 provinces (15,27-30). However, the implementation of national cancer screening programs for common cancers still encounters difficulties. The challenges we are facing include poor acceptance, low detection rate, high expenditure, shortage of qualified health care personnel and insufficient government funding. Most recently, Chinese government released the blueprint guide of "Healthy China 2030", in which an official goal was set to reduce premature mortality of major noncommunicable diseases by $30 \%$ from 2015 to 2030 . Since cancer is one of the most important noncommunicable diseases and the major cause of death in our country, improvement of the effectiveness of cancer prevention and control programs and policies plays a crucial role in achieving this health life indicator. 
The merit of this study is the large population coverage of the current cancer registry data. NCCRC has been publishing population-based cancer statistics of China annually since 2006. Along with the rapid increasement of cancer registries all over the country, population coverage of cancer registry data has been expanding constantly, resulting in an enhancement of representativeness of cancer registry data. On the other hand, the quality of cancer registry data still needs to be improved. In the current study, 339 (75.5\%) out of 449 cancer registries' data met the criteria of quality control. The proportion of acceptance was relatively high compared to previous years, but there was still room for improvement (10).

\section{Acknowledgements}

We thank the Bureau of Disease Control, National Health and Family Planning Commission of the People's Republic of China for their support to this study. We gratefully acknowledge all the population-based cancer registries for the effort they put in data collection, data sorting, data verification and database creation. The authors take full responsibility for analyses and interpretation of these cancer registry data. This study is supported by Ministry of Science and Technology (2014FY121100), and CAMS Innovation Fund for Medical Sciences (CIFMS) (201612M-2-004).

\section{Footnote}

Conflicts of Interest: The authors have no conflicts of interest to declare.

\section{References}

1. Torre LA, Bray F, Siegel RL, et al. Global cancer statistics, 2012. CA Cancer J Clin 2015;65:87-108.

2. Chen $\mathrm{W}$, Zheng R, Zhang S, et al. Cancer incidence and mortality in China, 2013. Cancer Let 2017;401:63-71.

3. National Cancer Center. Chinese Guideline for Cancer Registration. Beijing: People's Medical Publishing House, 2016.

4. Curade MP, Edwards B, Shin HR, et al. Cancer incidence in five continents. Volume IX. IARC Sci Publ 2008:1-837.

5. Ferlay J, Soerjomataram I, Dikshit R, et al. Cancer incidence and mortality worldwide: sources, methods and major patterns in GLOBOCAN 2012. Int $\mathrm{J}$ Cancer 2015;136:E359-86.

6. Siegel RL, Miller KD, Jemal A. Cancer statistics, 2018. CA Cancer J Clin 2018;68:7-30.

7. Chen $\mathrm{W}$, Zheng R, Zhang S, et al. Annual report on status of cancer in China, 2010. Chin J Cancer Res 2014;26:48-58.

8. Chen $\mathrm{W}$, Zheng R, Zeng H, et al. Annual report on status of cancer in China, 2011. Chin J Cancer Res 2015;27:2-12.

9. Chen $\mathrm{W}$, Zheng R, Zuo T, et al. National cancer incidence and mortality in China, 2012. Chin J Cancer Res 2016;28:1-11.

10. Chen $\mathrm{W}$, Zheng R, Baade PD, et al. Cancer statistics in China, 2015. CA Cancer J Clin 2016;66:115-32.

11. Ferlay J, Shin HR, Bray F, et al. Estimates of worldwide burden of cancer in 2008: GLOBOCAN 2008. Int J Cancer 2010;127:2893-917.

12. Lee YC, Lin JT, Wu HM, et al. Cost-effectiveness analysis between primary and secondary preventive strategies for gastric cancer. Cancer Epidemiol Biomarkers Prev 2007;16:875-85.

13. Ma JL, Zhang L, Brown LM, et al. Fifteen-year effects of Helicobacter pylori, garlic, and vitamin treatments on gastric cancer incidence and mortality. J Natl Cancer Inst 2012;104:488-92.

14. Li WQ, Ma JL, Zhang L, et al. Effects of Helicobacter pylori treatment on gastric cancer incidence and mortality in subgroups. J Natl Cancer Inst 2014;106:dju116.

15. Wei WQ, Chen ZF, He YT, et al. Long-term followup of a community assignment, one-time endoscopic screening study of esophageal cancer in China. J Clin Oncol 2015;33:1951-7.

16. Lao-Sirieix P, Fitzgerald RC. Screening for oesophageal cancer. Nat Rev Clin Oncol 2012;9: 278-87.

17. Zeng $\mathrm{H}$, Zheng R, Guo $\mathrm{Y}$, et al. Cancer survival in China, 2003-2005: a population-based study. Int J Cancer 2015;136:1921-30.

18. Dwyer-Lindgren L, Mokdad AH, Srebotnjak T, et al. Cigarette smoking prevalence in US counties: 19962012. Popu Health Metr 2014;12:5.

19. The National Lung Screening Trial Research Team. Reduced lung-cancer mortality with low-dose computed tomographic screening. N Engl J Med 2011;365:395-409.

20. Moyer VA; U.S. Preventive Services Task Force. 
Screening for lung cancer: U.S. Preventive Services Task Force recommendation statement. Ann Intern Med 2014;160:330-8.

21. Li J, Zhang BN, Fan JH, et al. A nation-wide multicenter 10-year (1999-2008) retrospective clinical epidemiological study of female breast cancer in China. BMC cancer 2011;11:364.

22. Fan L, Zheng $\mathrm{Y}, \mathrm{Yu} \mathrm{KD}$, et al. Breast cancer in a transitional society over 18 years: trends and present status in Shanghai, China. Breast Cancer Res Treat 2009;117:409-16.

23. McPherson K, Steel CM, Dixon JM. ABC of breast diseases: Breast cancer-epidemiology, risk factors, and genetics. BMJ 2000;321:624-8.

24. Fan L, Strasser-Weippl K, Li JJ, et al. Breast cancer in China. Lancet Oncol 2014;15:e279-89.

25. Chen C, Sun S, Yuan JP, et al. Characteristics of breast cancer in Central China, literature review and comparison with USA. Breast 2016;30:208-13.

26. Wang $\mathrm{K}$, Ren $\mathrm{Y}$, Li $\mathrm{H}$, et al. Comparison of clinicopathological features and treatments between young ( $\leq 40$ years) and older ( $>40$ years) female breast cancer patients in West China: A retrospective, epidemiological, multicenter, case only study. PloS one 2016;11:e0152312.

27. Lu YF, Liu ZC, Li ZH, et al. Esophageal/gastric cancer screening in high-risk populations in Henan Province, China. Asian Pac J Cancer Prev 2014; 15:1419-22.

28. Zhou QH, Fan YG, Bu H, et al. China national lung cancer screening guideline with low-dose computed tomography (2015 version). Thorac Cancer 2015; 6:812-8.

29. Song QK, Wang XL, Zhou XN, et al. Breast cancer challenges and screening in China: lessons from current registry data and population screening studies. Oncologist 2015;20:773-9.

30. Di J, Rutherford S, Chu C. Review of the cervical cancer burden and population-based cervical cancer screening in China. Asian Pac J Cancer Prev 2015; 16:7401-7.
Cite this article as: Chen $\mathrm{W}$, Sun $\mathrm{K}$, Zheng R, Zeng H, Zhang S, Xia C, Yang Z, Li H, Zou X, He J. Cancer incidence and mortality in China, 2014. Chin J Cancer Res 2018;30(1):1-12. doi: 10.21147/j.issn.1000-9604.2018.01.01 